\title{
THE RELIABILITY OF ULTRASONOGRAPHIC ASSESSMENT OF NEONATAL HIPS
}

\author{
J. J. DIAS, I. H. THOMAS, A. C. LAMONT, B. S. MODY, J. R. THOMPSON \\ From Leicester Royal Infirmary, England
}

Ultrasound scans were made of the hips of 209 neonates born consecutively over a two-week period. Of the 418 scans, 62 images were selected at random and 25 of these were duplicated to give a total of 87 scans.

These static images were then presented to five experienced observers who each made nine different assessments and measurements. Interobserver and intraobserver agreement was calculated and expressed as kappa values. Our results showed poor reliability on both counts.

J Bone Joint Surg [ Br] 1993; 75-B :479-82.

Received 21 May 1992; Accepted 4 September 1992

Ultrasonic examination of the infantile hip is widely taught and practised (Clarke et al 1985; Berman and Klenerman 1986; Jones and Powell 1990), using the basic methods developed by Graf and Schuler (1987), for the classification and management of dysplasia. These methods assume that ultrasound scans of the hip are reproducible, and that measurements made on the static ultrasound images are reliable.

We have investigated the second of these assumptions by determining the interobserver and intraobserver agreement on various assessments and measurements.

\section{PATIENTS AND METHODS}

Over a two-week period in February 1989, ultrasound scans of the hips were performed on 209 neonates born consecutively in the Maternity Unit of Leicester Royal Infirmary. Written parental consent was obtained. The $5 \mathrm{MHz}$ linear transducer of an Acuson 128 Ultrasonography Unit (Acuson Corporation, Mountain View, California, USA) was used to obtain static images in the modified Graf position, described by Clarke et al (1985).

J. J. Dias, MD, FRCS, Consultant Orthopaedic Surgeon

I. H. Thomas, MD, FRCS, Consultant Orthopaedic Surgeon

A. C. Lamont, FRCR, Consultant in Paediatric Radiology

B. S. Mody, MB BS, MS Orth, Registrar in Orthopaedics

J. R. Thompson, PhD, Lecturer in Epidemiology

Department of Orthopaedic Surgery, Leicester Royal Infirmary,

Infirmary Square, Leicester LEI SWW, UK.

Correspondence should be sent to Dr B. S. Mody

C) 1993 British Editorial Society of Bone and Joint Surgery $0301-620 X / 93 / 3525 \$ 2.00$
This involved scanning in the coronal plane with the hip flexed to $90^{\circ}$.

From the pool of 418 hip scans, 62 images were selected at random, and 25 of these were duplicated to provide a total of 87 images, among which 25 occurred twice. These 87 images were studied by five observers, all experienced in neonatal ultrasound scanning. Two were consultant paediatric radiologists and three were orthopaedic surgeons. On each scan, each observer made nine observations, which had been precisely defined at discussions before the study. Eight of the observations were those described by Graf and Schuler (1987) as necessary to classify the infant hip as normal, immature, eccentric or dislocated. The ninth measurement was a new one, for femoral head cover, and is described below. Observations These are illustrated by the diagrams in Figures 1 to 4 . 1) Promontory. The edge of the superior bony acetabular rim is represented by the point at which the flat surface of the ilium meets the acetabular cavity (Fig. 1). This was recorded as: a) well-contoured and angular or sharp; b) blunted, rounded or flattened; or c) scan of insufficient quality for comment.

2) Bony moulding. Bony moulding of the acetabulum (Fig. 1) was recorded as: a) good, showing a deep socket; b) poor, showing a shallow ill-defined socket; or c) no confident observation could be made.

3) Baseline shape. The shape of the ilium (Fig. 1) was recorded as: a) straight; b) convex; or c) concave.

4) Cartilaginous acetabular rim. This is represented by the poorly-echogenic area between the promontory and the echogenic labrum (Fig. 1). This was recorded as: a) echofree, showing as a dark triangle; b) echogenic, with a speckled grey appearance; or c) not adequately seen.

5) Shape of the cartilaginous acetabular rim. This was recorded as: a) a right-angled triangle; b) an isosceles triangle (Fig. 1); or C) uncertain.

6) Labrum. This is represented by the triangular echogenic structure extending laterally from the cartilaginous rim (Fig. 1), and was recorded as: a) well-defined; b) blurred; or c) could not be identified.

7) Alpha angle. This defines the bony acetabulum as an acute angle between the baseline and a line drawn from the echo-free triradiate cartilage gap in the acetabulum to the promontory (Fig. 2).

8) Beta angle. This defines the position of the labrum, 

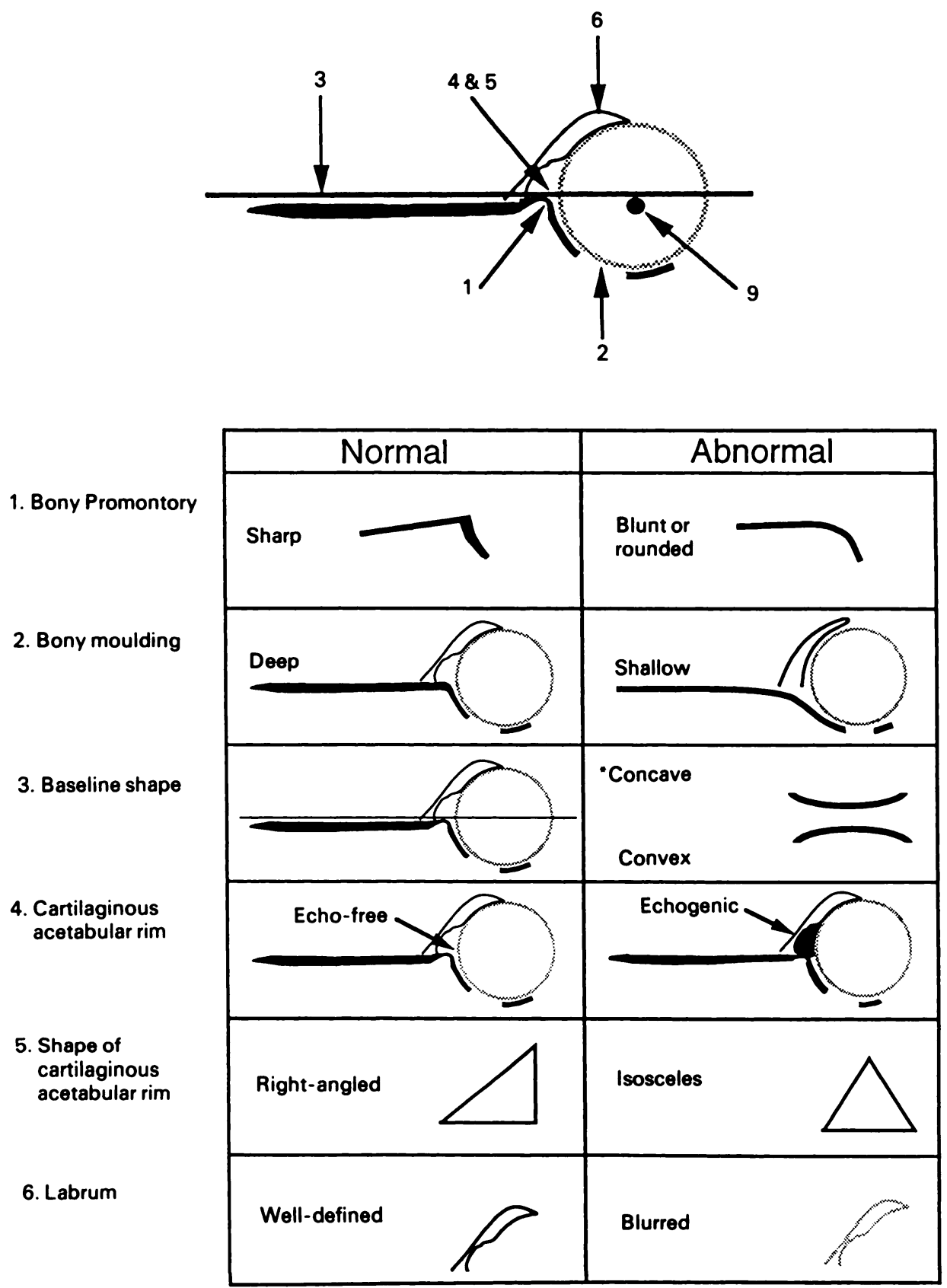

- invalidates the scan

Fig. 1

Diagrams of ultrasound scan images to show six of the parameters described in the text.

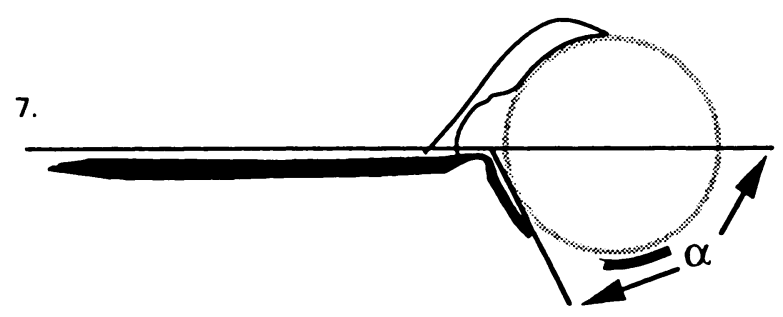

Fig. 2

Diagram to show the lines drawn to determine the alpha angle.

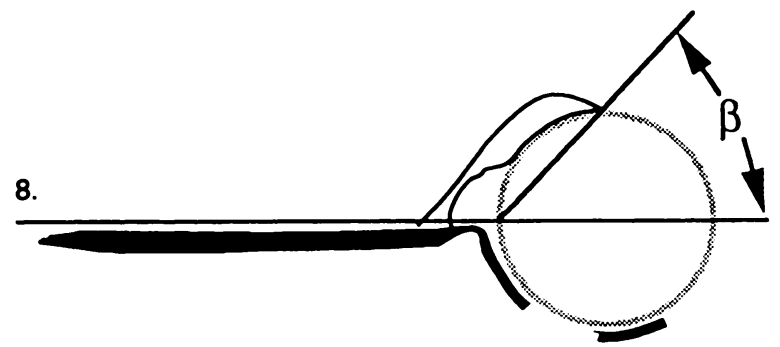

Fig. 3

Diagram to show the lines drawn to determine the beta angle. 
and is the angle between a line drawn from the tip of the labrum to the apex of the alpha angle and the baseline (Fig. 3).

9) Femoral head cover. A set of Mose's rings was superimposed on the fibrocartilaginous model of the femoral head to locate its centre (Fig. 4). The relationship of the centre of the femoral head and the baseline is recorded as: a) positive, (well covered), when the centre lay medial to the baseline; or b) negative, (poorly covered), when it lay lateral to the baseline (Fig. 1).

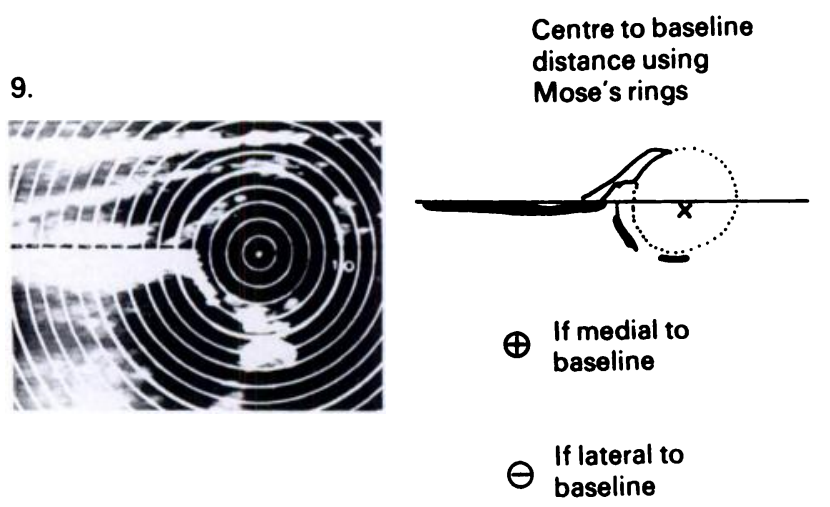

Fig. 4

Method of determining the centre of the femoral head for assessing the femoral head cover.

Table I. Intraobserver and interobserver agreement for seven categorical assessments on neonatal hip ultrasound scans. The results are expressed as average kappa values and as percentage of agreement. The standard deviation of the separate kappa estimates is given in parentheses

\begin{tabular}{|c|c|c|c|c|}
\hline & \multicolumn{2}{|c|}{ Intraobserver agreement } & \multicolumn{2}{|c|}{ Interobserver agreement } \\
\hline & $\begin{array}{l}\text { Average } \\
\text { kappa value }\end{array}$ & $\begin{array}{l}\text { Percentage } \\
\text { agreement }\end{array}$ & $\begin{array}{l}\text { Average } \\
\text { kappa value }\end{array}$ & $\begin{array}{l}\text { Percentage } \\
\text { agreement }\end{array}$ \\
\hline Promontory & $0.53(0.11)$ & 79 & $0.21(0.11)$ & 61 \\
\hline Bony moulding & $0.60(0.22)$ & 85 & $0.30(0.10)$ & 71 \\
\hline Baseline & $0.51 \quad(0.47)$ & 90 & $0.27(0.25)$ & 84 \\
\hline $\begin{array}{l}\text { Cartilaginous } \\
\text { acetabular rim }\end{array}$ & $0.68(0.19)$ & 84 & $0.21(0.09)$ & 59 \\
\hline $\begin{array}{l}\text { Shape of the } \\
\text { cartilaginous rim }\end{array}$ & $0.52(0.25)$ & 76 & $0.12(0.13)$ & 46 \\
\hline Labrum & $0.46(0.24)$ & 78 & $0.18(0.18)$ & 58 \\
\hline $\begin{array}{l}\text { Femoral head } \\
\text { cover }\end{array}$ & $0.55(0.21)$ & 83 & $0.09(0.38)$ & 61 \\
\hline
\end{tabular}

Table II. The intraclass correlations and limits of agreement for the two angular measurements

\begin{tabular}{lcc}
\hline & Intraobserver & Interobserver \\
\hline $\begin{array}{l}\text { Alpha angle } \\
\text { Intraclass } \\
\text { correlation }\end{array}$ & 0.69 & 0.65 \\
$\begin{array}{l}\text { Limits of } \\
\text { agreement }\end{array}$ & $+11.4^{\circ}$ & $+12.6^{\circ}$ \\
$\begin{array}{l}\text { Beta angle } \\
\text { Intraclass } \\
\text { correlation }\end{array}$ & 0.78 & 0.11 \\
$\begin{array}{l}\text { Limits of } \\
\text { agreement }\end{array}$ & $+14.9^{\circ}$ & $+19.0^{\circ}$ \\
\hline
\end{tabular}

Statistics. The readings made by each observer were compared as pairs with those of every other observer. For the categorical scales we used the unweighted kappa statistic to measure agreement (Fleiss 1981). Kappa can vary from negative values representing less than chance agreement, through 0 for chance agreement to +1 for perfect agreement. For each of the scales, the ten kappa statistics describing the extent of agreement between all pairings of the five observers were summarised by calculating their average and standard deviation.

For the two continuous measurements of alpha and beta angles we used the analysis of variance model suggested by Landis and Koch (1975) and the results were summarised by the intraclass correlation coefficients. We also calculated the limits of agreement as recommended by Bland and Altman (1986). The limits of agreement estimate the range within which $95 \%$ of the differences between two readings of the same scan can be expected to lie.

\section{RESULTS}

The agreement between observers on the seven observations which required categorical answers is shown in Table I. There was an unsatisfactory level of agreement between observers with regard to the bony promontory, the bony moulding, the baseline shape, the shape and appearance of the cartilaginous acetabular rim, the appearance of the labrum and the femoral head cover. The assessment of acetabular cover was not accurate enough to use this as a method of monitoring the development of the infant hip. As regards the 25 duplicate scans, the ability of each observer to reproduce his observations was better than the level of interobserver agreement, but was still insufficient to assess confidently serial changes in hip morphology (Table I).

For the two angular measurements (bony acetabular angle - alpha, and the labral angle - beta), the interobserver and intraobserver correlations between readings are shown in Table II. The measurement of the alpha angle had a fair degree of reproducibility between different observers and by the same observer. The betaangle measurements made by different observers showed very poor correlation, although single observers were able to reproduce their own previous measurement of this angle reasonably well.

These findings were confirmed by the limits of agreement method. The results were much wider for interobserver measurement of the beta angle than for intraobserver measurement, or the intra- and interobserver measurements of the alpha angle.

\section{DISCUSSION}

Ultrasound examination is a safe method of visualising the cartilaginous femoral head and can adequately define the acetabulum, thus overcoming some of the main 
drawbacks of radiography. Graf and Schuler's work has produced wide acceptance that a static scan can give a reliable image of the infant hip, and they have shown that deviations from the normal can be identified and that the degree of dysplasia can be quantified. They categorised the infant hip as normal, immature, eccentric or dislocated, and there is evidence that this method is satisfactory (Graf and Schuler 1987; Jones and Powell 1990). Engesaeter et al (1990), however, found that the standard ultrasound measurements did not correlate with the clinical and radiological outcome.

Our study suggests that agreement between observers is poor. The measurements on which Graf and Schuler based their classification are not reliable when made by different observers on the same scan, and were not very reproducible when repeated on the same scan by the same observer. Our results lead us to believe that measurements on static scans cannot be used with confidence either for screening or for monitoring treatment.

The interpretation of an ultrasound image relies on the recognition of boundaries and relationships of anatomical structures, and involves a subjective analysis of the distribution and texture of echo amplitudes (Shung 1985). The paths leading to decisions based on the observation of an image are complex; they may be prejudiced by previous experience, knowledge or lack of knowledge of the clinical situation, and many other factors such as adequacy of the image and the viewing conditions. Interobserver variation is common in medical work and affects results in both clinical and radiological studies. Intraobserver variation from day to day further complicates the issue. The classic error studies of Garland (1959) and Yerushalmy (1969) have explored these problems.

Despite our negative results for the value of static ultrasound scans, we believe that dynamic real-time ultrasound scanning is valuable in demonstrating laxity and reducibility. Engesaeter et al (1990) found that the dynamic assessment of stability was the only ultrasound technique which had a significant relationship to outcome.

It has been suggested that repeated clinical examinations may predispose to congenital dislocation of the infant hip (Moore 1989; Jones 1991). A single ultrasonic assessment may decrease the need for repeated examination, and this can be more gentle when a real-time image of the infant hip is available in addition to any proprioceptive perception of instability.

Conclusions. An ultrasound scan of a neonatal hip is an important additional method of assessment. It is much better than radiography in defining the cartilaginous femoral head and acetabulum and can be repeated with no additional risks. Dynamic screening shows movement and laxity of the hip, but conclusions should not be based only on measurements of static images; these appear to be unreliable.

No benefits in any form have been received or will be received from a commercial party related directly or indirectly to the subject of this article.

\section{REFERENCES}

Berman L, Klenerman L. Ultrasound screening for hip abnormalities: preliminary findings in 1001 neonates. $\mathrm{Br}$ Med J 1986; 293:719-22.

Bland JM, Altman DG. Statistical methods for assessing agreement between two methods of clinical measurement. Lancet 1986; i: 307-10.

Clarke NMP, Harcke HT, McHugh P, et al. Real-time ultrasound in the diagnosis of congenital dislocation and dysplasia of the hip. $J$ Bone Joint Surg [Br] 1985; 67-B:406-12.

Engesaeter LB, Wilson DJ, Nag D, Benson MKD. Ultrasound and congenital dislocation of the hip: the importance of dynamic assessment. J Bone Joint Surg [Br] 1990; 72-B:197-201.

Fleiss JL. Statistical methods for rates and proportions. 2nd ed. New York, etc: John Wiley \& Sons, 1981.

Garland LH. Studies on the accuracy of diagnostic procedures. AJR 1959; 82:25-38.

Graf R, Schuler P. Guide to sonography of the infant hip. New York: Thieme Medical Publishers Inc, 1987.

Jones DA. Neonatal hip stability and the Barlow test: a study in stillborn babies. J Bone Joint Surg [Br] 1991; 73-B:216-8.

Jones DA, Powell N. Ultrasound and neonatal hip screening: a prospective study of 'high risk' babies. J Bone Joint Surg [Br] $1990 ; 72-\mathrm{B}: 457-9$.

Landis JR, Koch GG. A review of statistical methods in the analysis of data arising from observer reliability studies (Part I). Statist Neerland 1975; 29:101-23.

Moore FH. Examining infants' hips - can it do harm? J Bone Joint Surg [Br] 1989; 71-B:4-5.

Shung KK. Ultrasonic characterisation of biological tissues. $J$ Biomech Eng 1985; 107:309-14.

Yerushalmy J. The statistical assessment of the variability in observer perception and description of roentgenographic pulmonary shadows. Radio Clin N Amer 1969; 7:381-92. 